\title{
Adaptive F.E. method for the shakedown and limit analysis of pressure vessels
}

Franco, J.R.Q. ${ }^{1}$, Ponter, A.R.S. ${ }^{2}$, Oden, J.T. ${ }^{3}$

1) UFMG, Escola de Engenharia, Departamento Estruturas, Belo Horizonte, MG, Brazil

2) Leicester University, Engineering Dept., Leicester, England

3) The University of Texasat Austin/TICAN, Austin, U.S.A.

ABSTRACT: This paper decribes a technique to calculate upper bounds estimates of limit and shakedown loads for pressure vessels by applying the Koiter's theorem. The flow law associated with an hexagonal prism yield surface, relates the plastic strain increments and curvatures to plastic multipliers. A suitable matrix also relates such a plastic strain field to a displacement field trough the classical relation. A novell method enforces a consistent relationship between nodal displacements and nodal plastic multipliers by minimizing the residual between the two independent description of the plastic increments, measured with respect to the energy norm. The shell was discretized into finite elements and the problem is then reduced to a minimization problem and solved by linear programming. A h-adaptive mesh refinement and the error indicator measured through the energy norm are used to improve accuracy of the solutions.

\section{INTRODUCTION}

Designing under conditions of high temperatures and large thermal transients has posed new and difficult problems of structural stability and integrity. In recent years with the advent of the Liquid Metal Fast Breeder Reactor and other new technologies a considerable effort has been devoted to studying these problems. The development of general numericall methods for strucutral analysis on the basis of shakedown and ratchetting concepts allows a more sophisticated treatment of such components.

The model problem can be described as the behaviour of an elastic-perfectly plastic body under a quasi-static process of cyclic deformation during collapse. The undeformed state of the body corresponds to an open bounded domain $\Omega \subset R^{3}$ (or volume $\Omega$ ) with smooth boundary or surface $S$ disjointed in two parts $S_{p}$ and $S_{u}$, i.e. $S=S_{u} \cup S_{p}$. The body is subjected to surface forces $k p_{i}$ prescribed on $S p$, where $k$ is a proportional loading parameter, and to body forces per unit volume $b_{i}$. Over the remainder of the total surface, $S_{u}$, applied surface displacement $u_{i}$ are also prescribed. In addition, a cyclic history of temperature $\theta(x, t)$ with a cyclic time $\Delta t$ may occur at a point $x$ within the domain $\Omega$, given by $\theta(x, t)=\theta_{0}+\theta(x, t)$ where $\theta_{0}$ denotes some reference temperature and $\theta(x, t)$ is a quasi static cycling temperature given by $\theta(x, t)=\Omega(x) \mu(t) \Delta \theta \quad 0<\mu<1 ; 0<\Omega<1$ and $\Delta \theta$ is the maximum temperature difference and $\Omega(x)$ is a non-dimensional shape function.

All the deformations are assumed to be sufficiently small so that changes in 
geometry can be disregarded.

\section{KOITER'S EXTENDED SHAKEDOWN THEOREM TO CYCLIC THERMAL LOADING}

(Franco 87) and (Franco and Ponter 93) described a formulation based upon the kinematical principles of Koiter's theorem (Koiter 56). The reduced problem was stated as follows (Franco, Ponter and Oden 94); for $k^{s} \geq k$ minimize

$k^{s}=\frac{\int_{0}^{T} d t \int_{\Omega}\left[\sigma_{i j}^{c}(t)-\hat{\sigma}_{i j}^{\theta}(x, t)\right] \epsilon_{i j}^{c}(t) d \Omega}{\int_{0}^{T} d t \int_{S_{p}} p_{i} u_{i}^{c} d S}$

The yield conditions governing the material plastic behaviour for thin shells was proposed by (Drucker 53) and (Drucker and Shield 58), which assumes that meridional curvature occurs only at nodal points and the meridional and circumferential membrane strains occur within the element with no change in curvature. A finite number of yield functions $\phi_{k}=\phi_{k}\left(N_{\phi}, N_{\theta}, M_{\phi}\right) \leq 0$ for $=1,8$ is needed to describe the linearized yield surface. The elastic domain is defined by the negative values of all yield functions and a zero value of one or more yield functions represents a state of stress on the yield surface.

The plastic strains and curvature are related to the generalized stresses by the flow rule, associated with such yield surface, in the form:

$\epsilon_{i j}^{c}=\sum_{k=1}^{6} \lambda_{k} \frac{\partial \phi_{k}}{\partial \sigma_{i j}}$ between hinges; $\kappa_{i j}^{c}=\theta_{k} \frac{\partial \phi_{k}}{\partial \sigma_{i j}}$ at hinges circles

where $\lambda_{k}$ are positive plastic multipliers and $\theta_{k}$ are positive rotations induced by the plastic unrestricted curvatures rates at the hinge circles. The minimization problem stated by Eq. 2.1 can only be solved for practical problems by the resort of finite element techniques.

\section{THE DISCRETIZED SHELL}

The finite element formulation for the elastic-plastic problem of thin shells may be derived from suitable interpolation of the displacement field and of the plastic multipliers as function of nodal values. For this purpose, let the shell be discretized into NE finite elements. The displacement field within the $i^{\text {th }}$ element may be expressed in terms of nodal global displacements $\left\{U_{N}\right\}^{i}$. The element nodal displacements $\left\{U_{N}\right\}^{i}$ can be divided in a constant rigid body motions $\left\{U_{0}^{i}\right\}$ which gives no contribution to the strain field and the so called natural displacements which generates the straining modes $\left\{U_{n}^{i}\right\}$. Thus $\left\{U_{e}^{i}(s)\right\}=\left\{U_{0}^{i}\right\}+\left[\Omega^{i}(s)\right]\left\{U_{n}^{i}\right\}$ where $\left[\Omega^{i}(s)\right]$ is a matrix of suitably chosen interpolation functions which ensures the continuity between adjacent elements when the assemblage is performed. The local displacement components normal $w(s)$ and tangential $u(s)$ to the meridional direction can be obtained by simple transformation. No effect of $M_{\theta}$ on the circumferential curvature is considered as discussed in (Drucker 53), (Drucker and Shield 58), (Onat 55), giving rise to three strain components from the strain-displacement relationship.

$$
\left\{\epsilon_{i j}^{1}(s, \phi)\right\}^{i}=\left\{\begin{array}{lll}
\epsilon_{\phi}^{1} & \epsilon_{\theta}^{1} & \kappa_{\phi}^{1}
\end{array}\right\}^{-1}
$$


The classical relation independent of the rigid body motions, for points within the element only, is now

$$
\left\{\epsilon_{i j}^{1}(s, \phi)\right\}^{i}=\left\{\begin{array}{c}
\epsilon_{\phi}^{1} \\
\epsilon_{\theta}^{1}
\end{array}\right\}^{i}=[B]^{i}\left\{U_{n}^{i}\right\}
$$

where matrix $[B]$ is the strain matrix which defines the classical strain-displacement relationship. The change in curvature $\kappa_{\phi}^{1}$ is considered to be localized at the plastic hinge circles.

The associated flow law relates the plastic strains to the plastic multipliers via a suitable matrix $[N]$. Assuming no variation of strain through the shell thickness the relations between plastic strains and plastic multipliers can be defined as follows:

$$
\left\{\epsilon_{i j}^{2}(s)\right\}^{i}=\left\{\begin{array}{c}
\epsilon_{\phi}^{2} \\
\epsilon_{\theta}^{2}
\end{array}\right\}^{i}=[N]\left\{\lambda_{k}^{i}(s)\right\} \quad \text { for } k=1,6
$$

Similarly to the displacement field, the plastic multipliers field for the element can be interpolated in terms of nodal parameters in a finite element approximation $\left\{\lambda_{k}^{i}(s)\right\}=\left[\Lambda^{i}(s)\right]\left\{\lambda_{k}^{n}\right\}$ where the only restrictions on $\lambda_{k}^{i}(s)$ is that it has to be non-negative. The reduction of the upper bound theorem (Equation 2.1) to a Linear Programming problem requires that the two separate description of the plastic increment given by Equations 3.4 and 3.5, must be consistent with each other at both nodes and within the element. The theory of conjugate approximations suggested by (Oden and Brauchli 71) will be used to obtain a consistent relationship between nodal plastic multipliers and displacements. The procedure is discussed in the following section.

\section{THE THEORY OF CONJUGATE APPROXIMATION}

Let the behaviour of individual elements be described in terms of nodal variables through a suitable interpolation matrix $[\phi]$ to give $\left\{\sigma_{i j}^{i}\right\}=\left[\phi_{i j}^{i}\right]\left\{\bar{\sigma}_{i}\right\}$ The biorthogonality condition from the theory of conjugate approximation, by (Oden and Brauchli $71)$ is satisfied if $\left[\phi_{i j}^{i}\right]=\left[R^{i}\right][H]^{i}$, where $[H]^{i}$ is a symmetric and non-singular matrix defined as $[H]^{i}=\left\{\int_{\Omega}\left[B^{i}\right]^{T}\left[R^{i}\right] d \Omega\right\}^{-1}$ Matrix $[R]$ has the same size of $[B]$ and its components are a set of functions obtained by a standard Galerkin procedure.

\section{THE ERROR MEASURE}

One of the most meaningful norm to estimate the error of a finite element solution is the energy norm defined by

$I=\|e\|^{2}=\int_{\Omega}\left\{\sigma_{i j}\right\}^{T}\left\{e_{\epsilon}\right\} d \Omega=\int_{\Omega}\left\{e_{\epsilon}\right\}^{T} D\left\{e_{c}\right\} d \Omega$

for $\left\{e_{\epsilon}\right\}=\epsilon_{i j}^{1}-\epsilon_{i j}^{2}$. Minimization of the error can be achieved by taking the derivative $\frac{d I}{d \bar{\sigma}}=0$ which gives the consistent relationship $\left\{U_{n}\right\}=[L]\left\{\lambda_{k}^{n}\right\}$ where $[L]=[H]^{T}\left\{\int_{\Omega}[R]^{T}[K(s)] d \Omega\right\}$ and $[K(s)]=[N][\Lambda(s)]$. This relationship also implies the condition $\int_{\Omega}[R]^{T}\left(\left\{\varepsilon_{1}\right\}-\left\{\varepsilon_{2}\right\}\right) d \Omega=0$ 
Such a condition requires that the difference between $\left\{\epsilon_{i j}^{1}\right\}$ and $\left\{\epsilon_{i j}^{2}\right\}$ shall be orthogonal to the arbitrary matrix $[R]$. The elements of $[R]$ need, therefore, to be chosen so that the strain difference is as small as possible. The components of $[R]$ can now be seen to be a set of functions in a Galerkin type procedure for the minimization of the strain differences. The final choice was as follows

$[R]=\left[\begin{array}{ccc}1 & 0 & 0 \\ 0 & \left(1-s / \ell_{i}\right) / R_{2} & \left(s / \ell_{i}\right) / R_{2}\end{array}\right]$

This choice implies that

$$
\begin{aligned}
& \int_{\Omega}\left(\varepsilon_{\phi}^{1}-\varepsilon_{\phi}^{2}\right) d \Omega=0 \\
& \int_{\Omega}\left(1-s / \ell_{i}\right)\left(\varepsilon_{\theta}^{1}-\varepsilon_{\theta}^{2}\right) / R_{2} d \Omega=0 \\
& \int_{\Omega} s / \ell_{i}\left(\varepsilon_{\theta}^{1}-\varepsilon_{\theta}^{2}\right) / R_{2} d \Omega=0
\end{aligned}
$$

i.e., $\left(\epsilon_{\phi}^{1}-\epsilon_{\phi}^{2}\right)$ is zero in the mean within each element and Eqs. 5.9 and 5.10 are equivalent to enforce the orthogonalities of $\left(\epsilon_{\theta}^{1}-\epsilon_{\theta}^{2}\right)$ to linear functions

These quantities are used to measure the local error within the element and have proved to be a very good error indicator. An $h$ adaptive mesh refinement on the basis of these error indicators allowed the improvement of the solutions giving rise to better shakedown limits and more exact mechanism of collapse.

\section{FINITE ELEMENT ALGORITHM}

The extended upper bound theorem was defined in Section 2 and can be formulated for thin axisymmetrical shells in the same form of Eq. 2.1. Assuming that $k$ is the optimal shakedown or limit load factor, the cost function Eq. 2.1 can be scaled by assuming that $\int_{S} p_{i} U_{i}^{c} d S=1$, which adds to the problem as a global constraint involving all the finite elements. The same problem can now be formulated as; for $k^{s} \geq k$ minimize

$k^{s}=\int_{0}^{T} d t \int_{\Omega}\left[\sigma_{i j}^{c}(t)-\hat{\sigma}_{i j}^{\theta}(x, t)\right] \epsilon_{i j}^{c}(t) d \Omega$

In addition to the global constraint, the optimization problem is subjected to the following constraints:

$$
\begin{aligned}
& \{u\}=\text { constant } \quad \text { on } S_{u} \quad \text { (BoundaryConditions) } \\
& \left\{\epsilon_{i j}\right\}=[B]\{U\} \quad \text { (Compatibility Conditions) } \\
& \left\{\epsilon_{i j}^{c}\right\}=[N]\{\lambda\} \quad \text { (Flow Law) }
\end{aligned}
$$

where $\{\lambda\}>0$

A major set of nodal constraints for the minimization problem, is defined as $\theta_{i}=U_{i}^{\prime(-)}+U_{i}^{\prime(+)}$ representing unrestricted curvatures(rotations) at each node. This allows the separation of bending at the nodes from membrane behaviour within the element when the shell is discretized. 


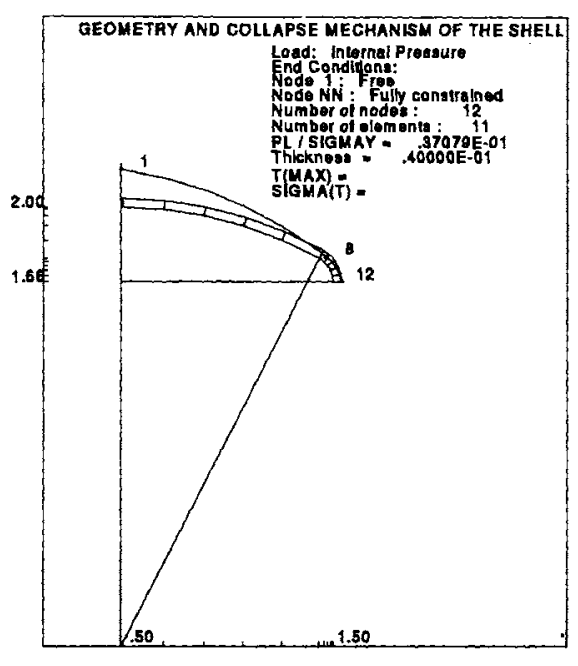

(a) Solution-Collapse Mechan. - NonOptimized Mesh

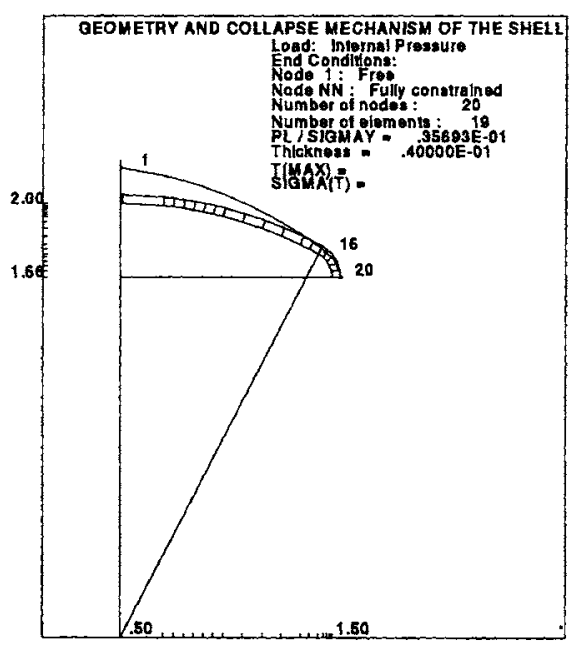

(c) Solution-Collapse Mechan.- Optimized Mesh

Figure 1: Solutions for a Torispherical Head

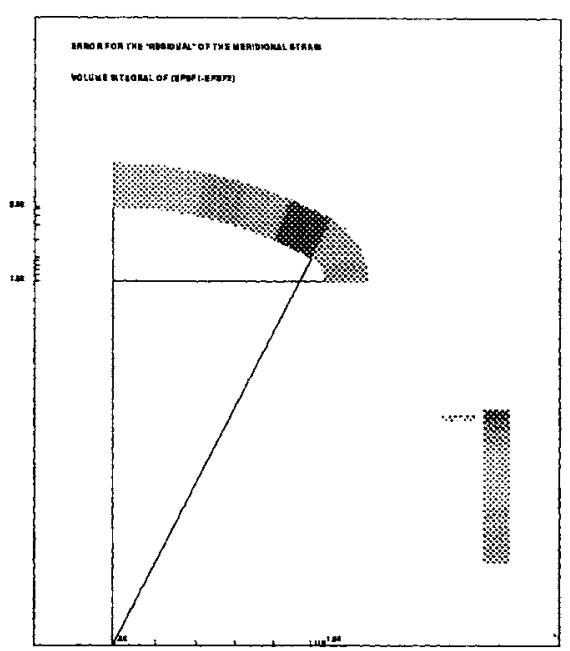

(b) Error Distribution

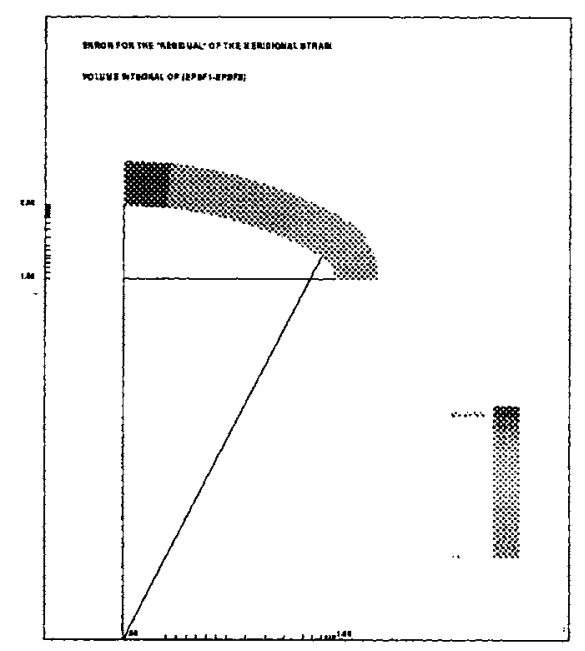

(d) [Error Distribution 


\section{NUMERICAL SOLUTIONS}

Four basic axisymmetrical elements, cylindrical, conical, spherical and toroidal, has been individually used with the technique. The following solutions represent the combination of two types of elements used to construct a torispherical head. The limit loads and corresponding collapse mechanisms for a non-optimized and a optimized mesh are presented respectively in Figs. 1(a) and 1(c) for a particular geometry. Figs. 1(b) and 1(d) show the error distributions within the elements for each case, measure with respect of one of the error indicator (Eq. 5.8) discussed in Section 5 . The optimized solution was obtained by adaptively refining the mesh.

\section{CONCLUSIONS}

A general technique for the limit and shakedown analysis of pressure vessels has been presented. A consistent relationship between the kinematically admissible displacement field and the pure plastic strain increments was enforced through the minimization of the residual between the two independent description of the plastic strain increments described in terms of nodal displacement and of nodal plastic multipliers. The estimate of such error measured with respect of the energy norm was used to improve the solutions.. The minimization procedure induced the strain residual in the meridional direction to be zero in the mean whereas a much stronger condition was induced in the circumferential direction where the residual is orthogonal to arbitrary linear functions. Orthogonality of the strain residual to arbitrary linear functions in both directions will be explored in future work.

\section{REFERENCES}

Drucker, D. C., "Limit Analysis of Cylindrical Shells Under Axially Symmetric Loading," Proc. 1st Midwest. Conf. Solids Mech., Urbana, IL, pp. 158163, 1953.

Drucker, D. C., and Shield, R. T., "Limit Analysis of Symmetrically Loaded Thin Shells of Revo-lution," J. Appl. Mech., Vol. 25, Trans. ASME 1958.

Franco, J.R.Q., "Bounding Techniques in Shakedown and Ratchetting," Ph.D. dissertation, University of Leicester, January 1987.

Franco, J.R.Q and Ponter A.R.S. 'A Displacement Formulation for the Finite Element Limit and Shakedown Analysis of Pressure Vessesl", The Congress on Structural Optimization, COPPE - UFRJ, Rio de Janeiro / August, 1993.

Franco, J.R.Q, Ponter A.R.S and Oden, J.T..'Adaptive F.E. Method for the Computation of Shakedown and Limit Load Problems of Axisymmetrical Shells 'Anais do XV CILAMCE, EEUFMG, Belo Horizonte/MG, p. 1579-1588 Dec/94.

Koiter, W. T., "A New General Theorem on Shakedown of Elastic Plastic Structures", Proc. Konk. Ned. Akad. Wett., B. 59, 1956, pp. 24-34.

Oden, J. T., and Brauchli, H. J., "On the Calculation of Consistent Stress Distribution in Finite Element Approximations," Int. J. for Num. Meth. in Eng., Vol. 3, pp. 317325, 1971.

Onat, E. T., "The Plastic Collapse of Cylindrical Shells Under Axially Symmetrical Loading," Quart. of Appl. Math., Vol. 13, pp. 6372, 1955.

Ponter, A.R.S., Karadeniz, S. and Carter, K. F., The Computation of Shakedown Limits for Structural Components Subjected to Variable Thermal Loading, Report EUR 12686, Directorate General, Science, Research and Development, Commission of the European Communities, Brussels, 1990, Chapter W, p. 93. 